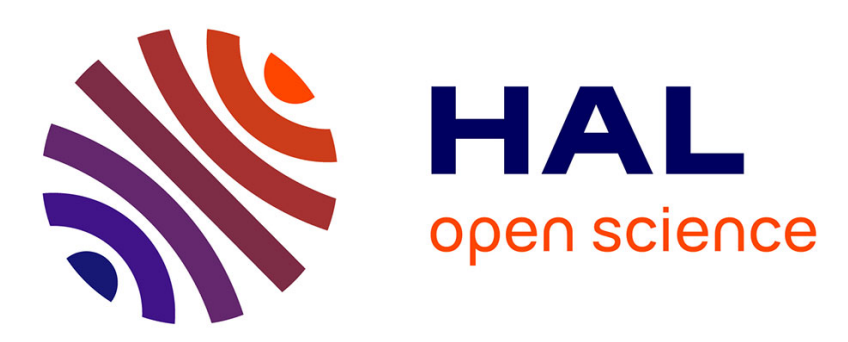

\title{
A Dynamic Distributed Algorithm for Read Write Locks (extended abstract)
}

\author{
Soumeya Leila Hernane, Jens Gustedt, Mohamed Benyettou
}

\section{To cite this version:}

Soumeya Leila Hernane, Jens Gustedt, Mohamed Benyettou. A Dynamic Distributed Algorithm for Read Write Locks (extended abstract). PDP 2012 - 20th Euromicro International Conference on Parallel, Distributed and Network-Based Processing, Feb 2012, München, Germany. pp.180-184, 10.1109/PDP.2012.32 . hal-00641068

\section{HAL Id: hal-00641068 \\ https://hal.inria.fr/hal-00641068}

Submitted on 15 Nov 2011

HAL is a multi-disciplinary open access archive for the deposit and dissemination of scientific research documents, whether they are published or not. The documents may come from teaching and research institutions in France or abroad, or from public or private research centers.
L'archive ouverte pluridisciplinaire HAL, est destinée au dépôt et à la diffusion de documents scientifiques de niveau recherche, publiés ou non, émanant des établissements d'enseignement et de recherche français ou étrangers, des laboratoires publics ou privés. 
A Dynamic Distributed Algorithm for

\section{Read Write Locks}

\section{(extended abstract)}

Soumeya Leila Hernane, Jens Gustedt, Mohamed Benyettou

RESEARCH

REPORT

$\mathrm{N}^{\circ} 7798$

November 2011

Project-Team AlGorille 



\title{
inzián
}

\section{A Dynamic Distributed Algorithm for Read Write Locks (extended abstract)}

\author{
Soumeya Leila Hernane誹, Jens Gustedt调, Mohamed Benyettou ${ }^{\ddagger}$ \\ Project-Team AlGorille
}

Research Report $n^{\circ} 7798$ - November 2011 - 12 pages

\begin{abstract}
In this paper, a new algorithm that extends Naimi-Trehel token-based mutual exclusion is proposed. Contributions are twofold.

First, our algorithm evolves within an changing environment; processes can join and leave the system while the parent tree is in ongoing transformation and, while requests accessing the critical section are inserted. Both the tree structure and the original algorithm are amended. We impose new rules and add new variables to the original structure, so as to maintain the connectivity of the parent tree as well as the Next chain, whatever circumstances.

Second, the possibility to share the token between processes is introduced. This allows either multiple concurrent readers or a single writer to enter the critical section. In the Next chain of successive readers, a newly introduced Reading handler ensures that all following requesters for read access may enter the critical section simultaneously, and keeps the token as long as at least one reader is in the critical section. In all cases, our approach can be implemented such that it keeps an average complexity of $O(\log (n))$ messages per request.
\end{abstract}

Key-words: Distributed locks, read-write locks, dynamic locking

\footnotetext{
* This article is accepted for publication in the proceedings of PDP 2012

${ }^{\dagger}$ LORIA, Nancy, France

¥ University of Science and Technology, Oran, Algeria

$\S$ INRIA Nancy - Grand Est, France 


\section{Un algorithme pour la gestion dynamique de verrous lecture/écriture (résumé étendu)}

Résumé : Nous proposons dans ce papier une extension de l'algorithme d'exclusion mutuelle, à base de jeton de Naimi-Tréhel. Notre contribution se présente sous deux angles différents.

Premièrement, notre algorithme progresse dans un environnement inconstant. Les processus peuvent joindre et quitter le système. En même temps, l'arbre parent subit des transformations au fur et à mesure que les requêtes d'accès à la section critique sont insérées. Les modifications portent aussi bien sur l'algorithme que sur la structure arborescente parent ainsi que sur la chaîne Next.

De ce fait, Nous imposons de nouvelles règles et de nouvelles variables aux structures de départ, de sorte que la connexité de l'arbre parent ainsi que celle de la chaîne Next soient maintenues.

Deuxièmement, nous rendons possible l'agencement du jeton partagé à l'exclusif. Ainsi, la section critique devient accessible, soit par plusieurs lecteurs concurrents soit par un seul écrivain. Dans la chaîne Next. le gestionnaire des lecteurs est introduit pour assurer l'entrée en section critique de tous les lecteurs successifs. De même, le gestionnaire des lecteurs garde le jeton partagé tant qu'au moins un lecteur est en section critique.

Dans tous les cas de figure, l'implantation de notre approche garantit une complexité logarithmique de l'ordre de $O(\log (n))$ messages par requête.

Mots-clés : verrou repartie, verrous lecture/écriture, verrouillage dynamique 


\section{Introduction and Overview}

Several protocols have been proposed in the literature to solve mutual exclusion problems within distributed systems. They can be either permission-based (Lamport [1], Maekawa [2], Ricart-Agrawala [3]) or token-based (Naimi-Trehel [4], Raymond [5]). The first set allows entering the critical section (CS) after receiving permission from other nodes. They incur a high communication overhead. The second set conditions the entrance into the CS by the possession of a token which is passed between nodes. They differ from each other in the way the request messages are routed to reach the token. This group of algorithms is tree-based and many of them exhibit a $O(\log (N))$ complexity in terms of the number of messages per request. Our work focuses on this class of algorithms for the sake of this message complexity.

The distributed algorithm of Naimi-Trehel [4] based on path reversal is the benchmark for mutual exclusion. In this paper, we will merely refer to it in the version of Naimi-Trehel-Arnold [6], that additionally provides the proofs for the properties that we will use. Many other extensions of this algorithm have been proposed in the literature, we mention a few of them. A Fault tolerant token based mutual exclusion algorithm using a dynamic tree was presented by Sopena [7]. It improves over NaimiTrehel [4] by ensuring a lower cost in terms of messages in the presence of failures. Quinson-Vernier [8] provide a byte range asynchronous locking of the Naimi-Trehel algorithm based on sub-queues when partial locks are requested. Wagner-Mueller [9] have proposed token based read-write locks for distributed mutual exclusion. The protocol distinguishes read and write requests and presents good performance. However, up to our knowledge it has no proof of safety and liveness. In [10], Mohamed \& Naimi have proposed a solution of Readers/Writers in the distributed systems. A specification of the algorithm was given by predicates used to express the axioms and in order to prove the algorithm. However, the complexity has not been studied. Based on the same assumptions as the original algorithm of Naimi-Trehel, this work presents an extended algorithm. Gustedt's $\mathrm{DHO}$ [11] presents a theoretical programming paradigm and an interface, for locking and mapping data in both read and write modes. It neither provides nor forces any particular algorithm or protocol read-write locks that would be used by that abstract interface.

Contributions in this paper are twofold. First, we extend the original Naimi-Trehel algorithm so that the parent tree and the Next chain (for definitions of these terms please see the original Naimi-Trehel algorithm [10]) evolve in a dynamic environment, while maintaining safety and liveness properties, see Section 2 Second, in addition to exclusive locks hold by a single writer, we allow multiple readers to enter the CS, so that the algorithm covers both exclusive and concurrent locks, Section 3 . In this framework, we propose a new data structure that handles additional features. Finally, in Section 4 we conclude, in particular to advertise the use of this approach within an API that handles resources in large scale distributed systems.

\section{Mutual exclusion algorithm with volatile processes}

The Naimi-Trehel algorithm is based on a distributed queue along which the token circulates, and on a distributed tree structure for queries. The query tree is rooted at the tail of the queue to allow to append new requests to the queue at any moment. The basics of the Naimi-Trehel algorithm can be found in [4]. 


\subsection{Concurrent requests}

Within Naimi-Trehel's algorithm, a given parent $p_{1}$ of two processes $p_{2}$ and $p_{3}$ can be queried simultaneously by both. In such case, if $p_{1}$ treats $p_{2}$ first, $p_{3}$ is put on hold and is disconnected temporarily from the tree. An example from [12] is shown in Fig. 1 Initially, Fig. 1(a), $p_{1}$ holds the token and $p_{3}$ claims the CS by sending a request to its parent. in turn, $p_{1}$ updates its parent and its Next to $p_{3}$, Fig. 1(b). Then, $p_{2}$ and $p_{5}$ claim the CS. They send request to $p_{1}$ and set forthwith their parent to null. So, $p_{1}$ points towards $p_{2}$ and forwards the request to $p_{3}$, Fig. 1(c). Meanwhile, $p_{5}$ waits and is disconnected from the tree.

Once $p_{1}$ sent $p_{2}$ 's request to $p_{3}$, it switches to $p_{5}$ 's request. Thus, it forwards the request to $p_{2}$ and sets its parent to $p_{5}$. Meanwhile, $p_{2}$ is cut from the tree, Fig. 1(d)] In Fig. 1(e) $p_{2}$ 's request is achieved and $p_{5}$ 's ends in Fig. 1(f).

We notice that, processes set their parent variable to null as soon as they forward the request. Within a system of $n$ processes, $n-1$ processes may request the token concurrently and this will generate $n$ disjoint parent's trees. This example was presented in [12] in the context of node failures. In our proposal, processes handle one request at a time (Section2).

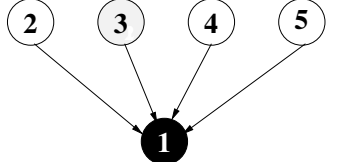

(a) initial state

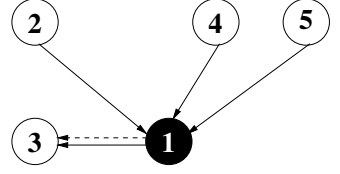

(b) $p_{3}$ requests the token

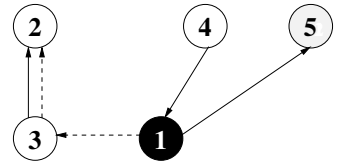

(e) $p_{5}$ in transit

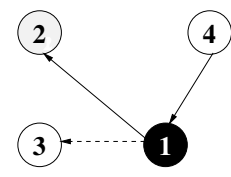

(c) $p_{2}$ and $p_{5}$ request the token

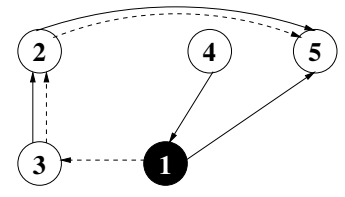

(f) completion of requests

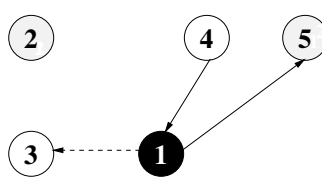

(d) $p_{2}$ and $p_{5}$ in transit

Figure 1: Example of concurrent requests of Naimi-Trehel's Algorithm [12]

We provide an extension of the algorithm that allows processes to enter or to leave the system. Note that if a process wants to enter the group, it just has to choose a relative parent to join the tree. It will then be connected to the system. So the difficulty in node dynamicity lies in the departure of processes, to which we thus focus in the sequel.

To that aim, we amend the original algorithm by requiring that processes are never cut from the parent tree, whatever the circumstances. This helps to keep all links alive if a given process leaves the system. We assume that a process does not support a new request before the previous one is completed.

\subsection{Atomic Operations}

In this subsection we amend the structure and query processing of the mutual exclusion algorithm, such that we avoid overlapping of operations that relate to a given process $p_{i}$. In order to meet new requirements of the extended algorithm, we add to the set of variables as defined above: 
Send: A Boolean that refers to the process state, initially set to false. If true, then the process has sent a request that is not completed.

Req: A list of received requests by a process, initially empty.

Wait_ack: A Boolean that refers to an acknowledgment (ACK) set by a given process, initially set to false.

Previous: Each process knows who will hold the token before him, it is easily updated simultaneously as Next. Next and Previous form a doubly linked list.

Let $\left\{p_{1} . . p_{i} . . p_{n}\right\}$ a finite set of processes within a distributed system. Processes are bound to states related to handling requests, not on the token possession. A process $p_{i}$ can be:

Idle: If it has a parent, it can either send a request to it and then switch Send to true, or receive a request from any other process that points on $p_{i}$ as parent. Req list is null.

Sending: $p_{i}$ sent a request that is not yet completed. It is not ready to receive any request as Send is true. $\operatorname{Req}$ is null.

Busy: $p_{i}$ handles and registers a request. It is not ready to register others, however it can put them in $\boldsymbol{R e q}$ list.

We consider a branch of the parent tree $\left\{. . p_{j-1}, p_{j}, p_{j+1} . . p_{n}\right\}$ as a sequence of processes such that $p_{n}$ is the root, whereas $p_{j+1}$ is the parent of $p_{j}$. Hence, $p_{j}$ points towards $p_{j+1}$. Suppose initially, $p_{0}$ is the root that holds the token.

As in the original Naimi-Trehel algorithm, assume that at a time $t, p_{j}$ requests the token from its parent $p_{j+1}$. Additionally we will always require that, before, it sets the Send variable to true.

Conversely, if $p_{j+1}$ has sent a request that is not yet achieved, it puts $p_{j}$ 's request on hold and stores it in $\boldsymbol{R e q}$ list. As soon as $p_{j+1}$ is available, it starts processing the $p_{j}$ 's request. If its parent exists $(j+2), p_{j+1}$ sends it to $p_{j}$ and waits for ACK. Then, $p_{j}$ resends the request to $p_{j+2}$.

Similarly, if $p_{j+2}$ is not busy, it sends its parent to $p_{j}$ and waits for ACK. Otherwise, it puts the pending request in $\boldsymbol{R e q}$ list. The processing is carried on until $p_{n}$ is claimed. Then, $p_{n}$ informs $p_{j}$ that it is the root of the tree and, it registers $p_{j}$ as the Next. In turn, $p_{j}$ sets its Previous to $p_{n}$. Processes $p_{j+1} . . p_{n}$ update their parent to $p_{j}$.

Suppose that $p_{j+1}$ is a parent of another process $p_{i}$. As in Figure $1, p_{1}$ is the parent of $p_{2}$ and $p_{5}$. Suppose that $p_{i}$ sends a request to $p_{j+1}$. Knowing that $p_{j+1}$ has not yet received an ACK from $p_{j}$, it puts the $p_{i}$ request in Req list.

The processing ends when $p_{j}$ sends an ACK to its parent $\left(p_{j+1}\right)$ and to all its ascendants into the parent's tree. It was then that processes $p_{j+1} . . p_{n}$ handle Next requests that are in $\boldsymbol{R e q}$ list, according to the FIFO policy. Then, $p_{j}$ sets its parent variable to null and its Send value to false.

Thus, we avoid the overlap of requests. Likewise, we ensure consistency in the logical order of requests. 


\subsection{Average Message Complexity}

The average number of messages needed for a request to reach a root within NaimiTrehel's algorithm depends on the height of the request tree process. By using the arguments from [6] we will show that this results in an average message complexity of $O(\log (N))$, where $N$ is the whole number of processes in the system.

In the original algorithm, a request is propagated through the parent tree from a son $p_{j}$ to parent $p_{j+1}$, as the requesting process $p_{j}$ sends, itself a message to each process in the parent tree. In our algorithm, in addition each process $\left(p_{j+1} . . p_{n}\right)$ in the branch of the tree, sends a message to the requesting process $p_{j}$ containing its parent. Then, from the end of the tail, $p_{j}$ sends acknowledgments to its parent and to all its ascendants. So in summary, the message complexity per request grows by 3 and will be remain $O(\log (N))$ on average, as in the original algorithm.

Notwithstanding, the extra cost of the modified algorithm does not depend of the whole number of processes and thus, remains $O(\log N)$. By this addition, we are aiming to provide a mutual exclusion algorithm in a dynamic environment.

\subsection{Formal Description}

There is no common clock in our system. However, we introduce a local clock to determine the sequence of events that happen at a given process $\rho$. Values are assigned to variables described above for each state of the process. By that, atomicity for requests is ensured, while overlap is avoided.

Let $\mathrm{T}=\left(t_{0}, \ldots, t_{n}\right)$ an ordered sequence of times with $t_{i}<t_{i+1}$ refering to request reception by $\rho$, and let $\mathrm{T}^{\prime}=\left\{\ldots, t_{i}^{\prime}, \ldots\right\}$ be the set of times of the acknowledgments of those registered requests. Note that $\rho$ doesn't record a new request until it received the ACK of the previous one. We have

$$
t_{0}<t_{0}^{\prime}<\cdots t_{i}<t_{i}^{\prime} \cdots<t_{n}<t_{n}^{\prime}
$$

Let State $(\rho, t)$ be the state of $\rho$ at time $t$. A process $\rho$ is in Idle state if it is neither sending, or receiving requests, nor waits for an ACK. If $\rho$ is Busy, it waits for an ACK from the process whose request has been registered. If $\rho$ is Sending, it has sent a request to its parent and has not finished sending ACKS. In summary, for each $t$ in $\left[t_{i}^{\prime}, t_{i+1}\right]$ the following hold:

$$
\begin{gathered}
\operatorname{STATE}(\rho, t)=\text { Idle } \Rightarrow\left\{\begin{array}{l}
\text { SEND }=\text { false } ; \text { REQ }=N u l l \\
\text { WAIT_ACK }=\text { false }
\end{array}\right. \\
\operatorname{STATE}(\rho, t)=\text { Busy } \Rightarrow\left\{\begin{array}{l}
\text { SEND }=\text { false } ; \text { REQ } \neq \text { Null } \\
\text { WAIT_ACK }=\text { true }
\end{array}\right. \\
\operatorname{STATE}(\rho, t)=\text { Sending } \Rightarrow\left\{\begin{array}{l}
\text { SEND }=\text { true } ; \text { REQ }=\text { Null } \\
\text { WAIT_ACK }=\text { false }
\end{array}\right.
\end{gathered}
$$

Also, $\rho$ must be in the Idle state before sending or recording a given request, i.e for $t_{1}<t_{2}$, if $\operatorname{State}\left(\rho, t_{1}\right)$ and $\operatorname{State}\left(\rho, t_{2}\right)$ are Sending or Busy, then there is an intermediate $t^{\prime}$ with $t_{1}<t^{\prime}<t_{2}$ such that $\operatorname{STATE}\left(\rho, t^{\prime}\right)=I d l e$. 


\subsection{A Dynamic Mutual Exclusion Algorithm}

We impose the condition that the tree still always connected. For that, we bring additional modifications that will be dealing with the exit strategy for processes. We add the following variables to the previous structure, to maintain the connectivity of the tree.

Child $[1 . . m]$ : An array allocated dynamically, containing $m$ process IDs that have $\rho$ as parent.

Del: Boolean, initially false. It is permanently set to true once $\rho$ leaves the system.

End_del: Boolean, initially false. It is set to true while $\rho$ is negotiating its departure, and goes back to false at the end of that process.

Several events occur before a process $\rho$ leaves the system. We summarize them in chronologically as follows:

1. If $\rho$ is in the CS, it leaves it. Then, it grants its Next if it exists and, this will be the new root.

2. $\rho$ broadcasts a message to its children. It asks each of them if it is also leaving, Sending or Busy. $\rho$ waits for a message from each of them, this will avoid deadlock.

3. If one of the children is now leaving the system, its End_del variable is true. Thus, $\rho$ waits until the End_del of the Child is false. It will receive a message subsequently.

4. Once all children are notified, they won't send any request or message to $\rho$, until they receive a new parent.

5. Once $\rho$ becomes Idle and its $\boldsymbol{R e q}$ list becomes empty, it sets Del and End_del to true.

6. If $\rho$ is the current root (and thus Next was empty in 1), then $\rho$ elects one of its children $R$ to become the new root.

7. $\rho$ sends either this new root $R$ or its parent to its children.

8. All children update their parent variable. Thus, the links of the tree are well maintained.

9. If they are set, $\rho$ notifies its Next and its Previous such that the doubly linked list can be shortcut by them.

10. At the end of the leaving process, $\rho$ sets End_del to false and, if it was non-null, notifies parent that it has finished the departing procedure.

By that, as the parent tree remains connected, the Next and the Previous chains links are preserved.

We note that Liveness and safety properties are maintained. The complete proof of these properties will be presented in the full version of this paper. 

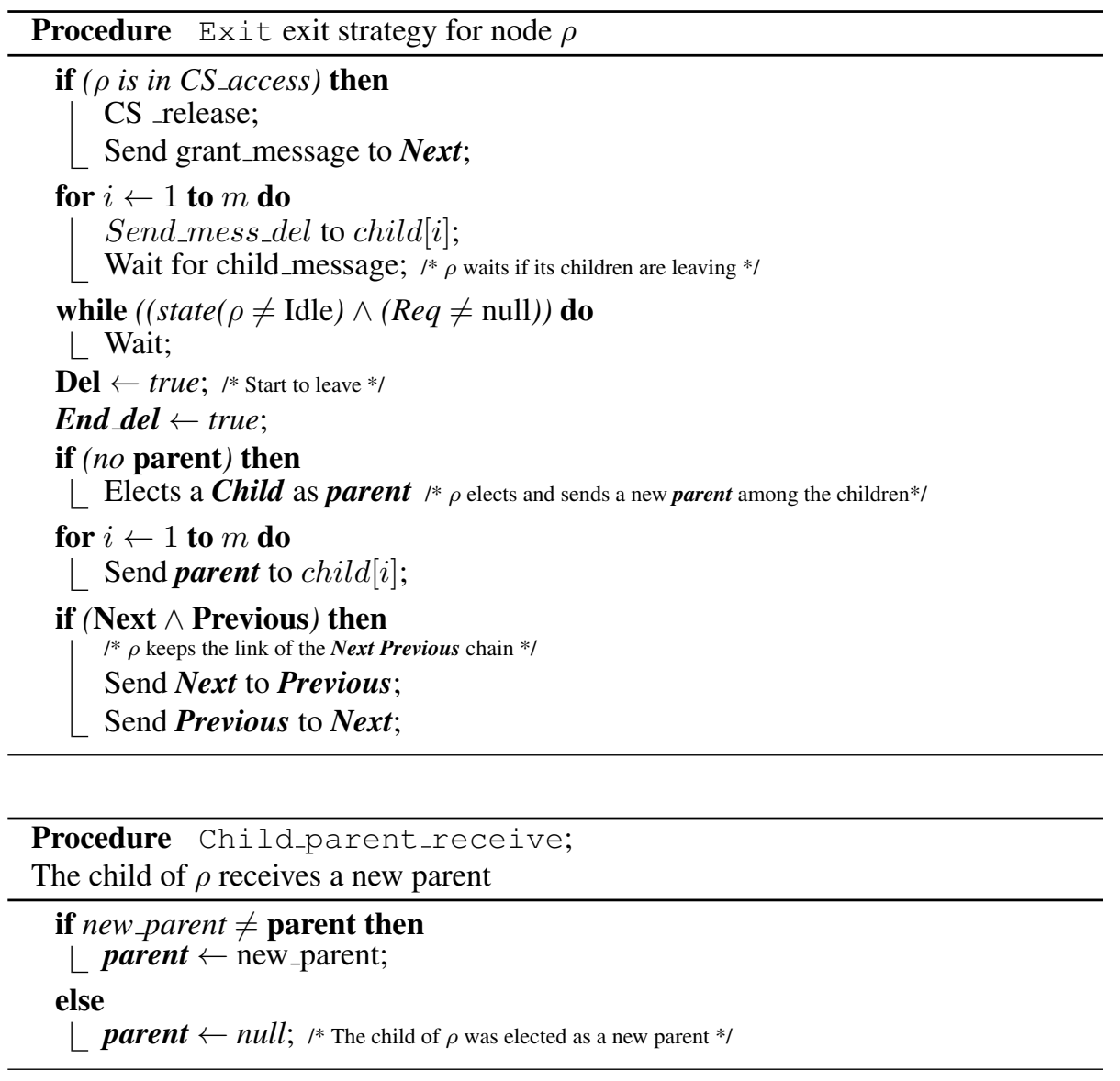

\section{Read Write Dynamic Algorithm}

This section relates to the second contribution in which the sharing of the token is introduced to the previous extended algorithm, resulting in a a read-write mutual exclusion algorithm with volatile processes. Contrary to the contribution of [9], in our approach we introduce a Reading handler of concurrent requests. It will cope with the volatility of processes. However, the exit strategy of the Reading handler will be dealt with later. First of all, we add the following local variables to the structure of a given process $\rho$, see Section 2.2.

Type: Request type, W (exclusive write) or R (inclusive read).

Reading handler: Referring to the first process that requests a shared token after an exclusive one. Initially set to null.

Reader counter: A counter that is incremented if $\rho$ is a Reading handler and at each subsequent read request. Set to zero, initially.

In the following, we assume that the reader chain is a Next chain of pending successive read requesters. Initial values are added within the initialization() function. 


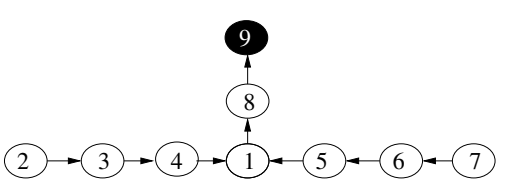

(a) $p_{9}$ holds the token

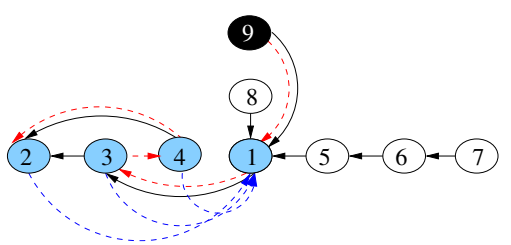

(c) $p_{3}, p_{4}$ and $p_{2}$ request the shared token

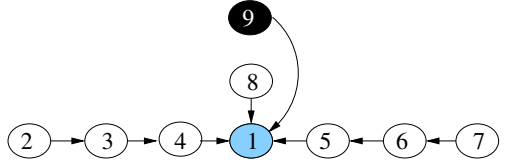

(b) $p_{1}$ requests the shared token

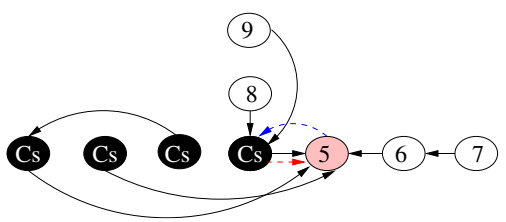

(d) The reading chain enter in $\mathrm{CS}$ and $p_{5}$ requests an exclusive token

$R$ request $\bigcirc$ Wrequest $\longrightarrow$ Parent $-\rightarrow$ Next $-\rightarrow$ Previous

Figure 2: Example of mixed requests

\subsection{Handling requests}

Within the basic algorithm of Naimi-Trehel, the root of the tree updates its Next to $\rho$ when the latter sends a request to its parent [4]. We focus on the maintenance of the Next chain. Assume a chain of processes $\left\{p_{0} . . p_{j-1}, p_{j}, p_{j+1} . . p_{n}\right\}$ that have pending requests and where $p_{j+1}$ is the Next of $p_{j}$ and $p_{j-1}$ is the Previous of $p_{j}$. At that time, $p_{j}$ and $p_{j-1}$ exchange their respective type. Then, $p_{j-1}$ sets its Next to $p_{j}$. Several cases may occur:

- If $p_{j-1}$ 's type is $\mathrm{W}$ and $p_{j}$ 's type is R then $p_{j}$ sets the Reading handler to itself, its Previous to $p_{j-1}$ and increments the Reader counter.

- If $p_{j-1}$ 's and $p_{j}$ 's type are R then, $p_{j}$ sends a message and sets its Previous to the Reading handler. This one increments the Reader counter. Note that the Reading handler can be within $\left[p_{0} . . p_{j-1}\right]$.

- If $p_{j-1}$ 's type is $\mathrm{R}$ and $p_{j}$ 's type is W then $p_{j}$ sets its Previous to the Reading handler. The latter sets its Next to $p_{j}$.

- If $p_{j-1}$ 's and $p_{j}$ 's type are W then they update their Next and Previous variable respectively, just as before in the case that we only maintained exclusive access to the CS.

Figure 2 shows an example of 4 consecutive pending requests. $p_{1}$ is the Reading handler of $p_{2}, p_{3}$ and $p_{4}$. Figure 2(c) summarizes result of events of read requests that have been launched consecutively by $p_{3}, p_{4}$ and $p_{2}$. In Fig. 2(d), the reader chain enters in the CS and $p_{5}$ sends a write request. In terms of complexity, three additional messages are required, two for the type exchange and one to send the identification of the Reading handler. Thus, the message complexity of handling requests remains $O(\log (N))$. 


\subsection{Entering the CS}

Within a chain $\left\{p_{0} . . p_{j-1}, p_{j}, p_{j+1} . . p_{n}\right\}$ of successive read requesters, as soon as $p_{0}$ (the Reading handler) accesses the CS, it invites its Next to do so, and so on until the last read requester $p_{n}$. When it enters the CS, each process in the reader chain sends a message to the Reading handler which in turn increments the Reader counter. So, we have only one additional message per participating process in terms of complexity.

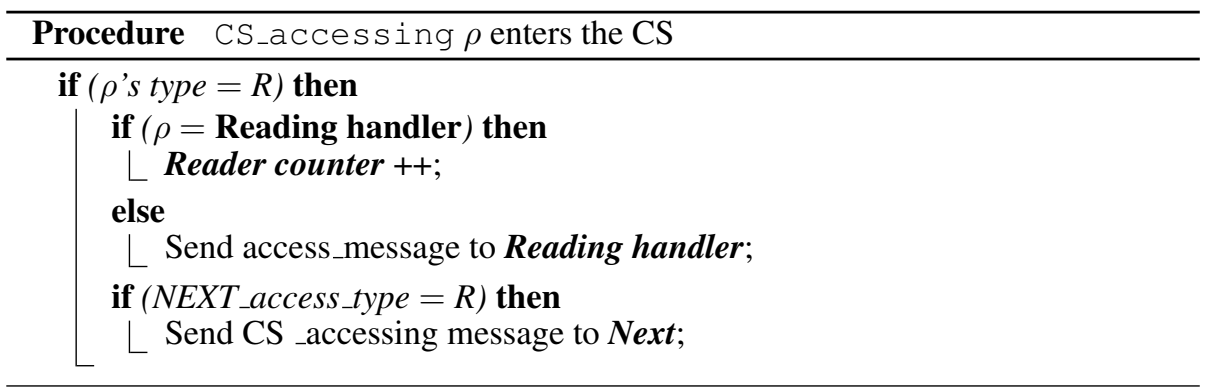

\subsection{Token release}

Once they release the token, processes send a message to the Reading handler, which decrements the Reader counter. We note that the previous of $p_{n+1}$ is the Reading handler $\left(p_{0}\right)$. The Reading handler doesn't release the token to the first process requesting an exclusive token following the reader chain, until the Reader counter is zero. Obviously, a process $\rho$ never shares an exclusive token with a shared one.

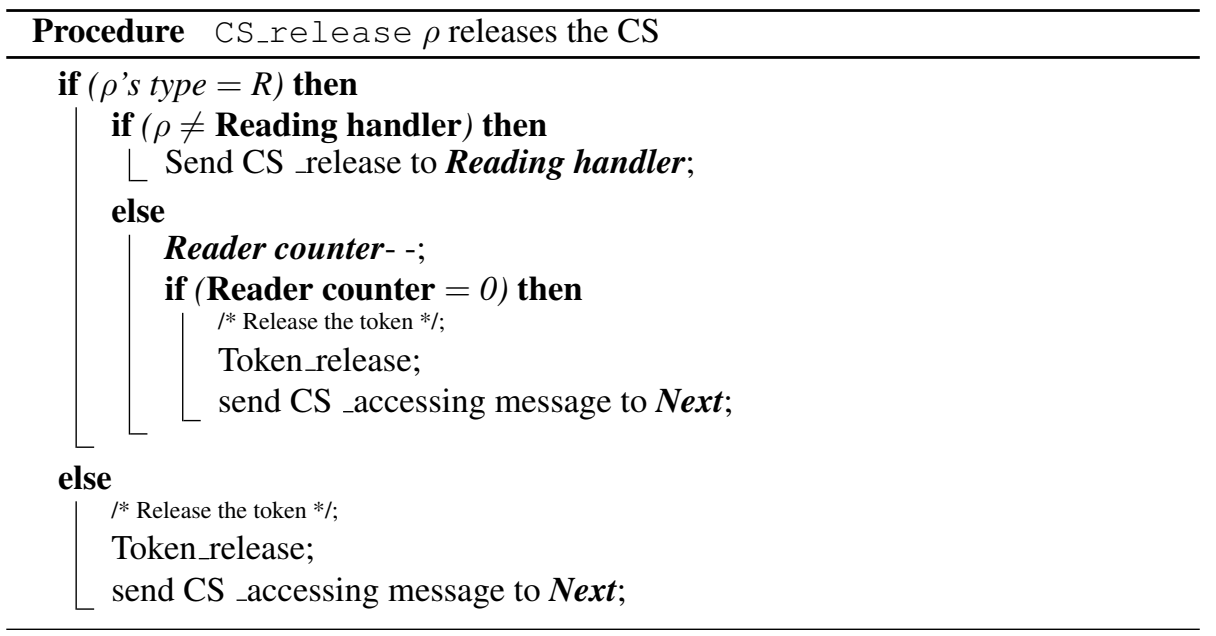

The correctness of the algorithm for the extension to shared tokens is will be available in the full version of this paper. 


\section{Conclusion}

A new approach of an extended model for a class of distributed mutual exclusion algorithms has been presented in this paper. Our model is an extension of the Naimi-Trehel token-based algorithm. It presents two main benefits; first, it allows a continuous evolution of dedicated distributed environment and secondly, it offers two different access modes to the CS. This contribution should reveal useful in context of grid computing, peer to peer computer networking or any large scale distributed infrastructure. In those systems, resources are set to disappear and reappear. The first modification of the algorithm provides this feature. Furthermore, our approach has a low (=logarithmic) complexity in expected number of messages per operation.

We are currently implementing our approach and plan to measure the ability of that algorithm to provide a robust and scalable framework such as grids. In ongoing work, we aim to join the current algorithm to an application programming interface, Data Handover $\mathrm{DHO}$ [11], which is a framework for an efficient management for locking and mapping data (an subranges of it) in both read and write modes. Both, our first and second modification of the Naimi-Trehel algorithm will support these ideas. The DHO functions allow the user to insert requests, to release resources and to leave the system, seamlessly to the internal structure of the algorithm provided in our previous work [13].

\section{References}

[1] L. Lamport, "Ti clocks, and the ordering of events in a distributed system," Commun. ACM, vol. 21, pp. 558-565, July 1978.

[2] M. Maekawa, "An algorithm for mutual exclusion in decentralized systems," ACM Trans. Comput. Syst., vol. 3, pp. 145-159, May 1985.

[3] G. Ricart and A. K. Agrawala, "An optimal algorithm for mutual exclusion in computer networks," Commun. ACM, vol. 24, pp. 9-17, January 1981.

[4] M. Naimi and M. Trehel, "How to detect a failure and regenerate the token in the $\log (N)$ distributed algorithm for mutual exclusion," in Proceedings of the 2nd International Workshop on Distributed Algorithms. London, UK: SpringerVerlag, 1988, pp. 155-166.

[5] K. Raymond, "A tree-based algorithm for distributed mutual exclusion," $A C M$ Transactions on Computer Systems, vol. 7, pp. 61-77, 1989.

[6] M. Naimi, M. Trehel, and A. Arnold, "A $\log (N)$ distributed mutual exclusion algorithm based on path reversal," J. Parallel Distrib. Comput., vol. 34, pp. 1-13, April 1996.

[7] J. Sopena, L. B. Arantes, M. Bertier, and P. Sens, "A fault-tolerant token-based mutual exclusion algorithm using a dynamic tree." in Euro-Par'05, 2005, pp. 654-663.

[8] M. Quinson and F. Vernier, "Byte-range asynchronous locking in distributed settings," in 17th Euromicro International Conference on Parallel, Distributed and network-based Processing - PDP 2009, Weimar, Germany, 2009. [Online]. Available: http://hal.inria.fr/inria-00338189/en/ 
[9] C. Wagner and F. Mueller, “Token-based read/write-locks for distributed mutual exclusion," in Proceedings from the 6th International Euro-Par Conference on Parallel Processing, ser. Euro-Par '00. London, UK: Springer-Verlag, 2000, pp. 1185-1195.

[10] A. Mohamed and B. Naimi, "Distributed concurrent control with readers and writers," in International Conference "PARALLEL 90", Springer Verlag, Southampton United Kingdom, 1990.

[11] J. Gustedt, "Data handover: Reconciling message passing and shared memory," in Foundations of Global Computing, ser. Dagstuhl Seminar Proceedings, J. L. Fiadeiro, U. Montanari, and M. Wirsing, Eds., no. 05081, Dagstuhl, Germany, 2006. [Online]. Available: http://drops.dagstuhl.de/opus/volltexte/2006/297

[12] M. Naimi and M. Trehel, "How to detect a failure and regenerate the token in the $\log (N)$ distributed algorithm for mutual exclusion," in WDAG, ser. Lecture Notes in Computer Science, J. van Leeuwen, Ed., vol. 312. Springer, 1987, pp. 155-166.

[13] S. Hernane, Leila, J. Gustedt, and M. Benyettou, "Modeling and Experimental Validation of the Data Handover API," in Advances in Grid and Pervasive Computing, ser. LNCS, Riekki, J., Ylianttila, M., Guo, and M., Eds., vol. 6646. Oulu, Finland: Springer, May 2011, pp. 117-126. [Online]. Available: http://hal.inria.fr/inria-00547598/en 


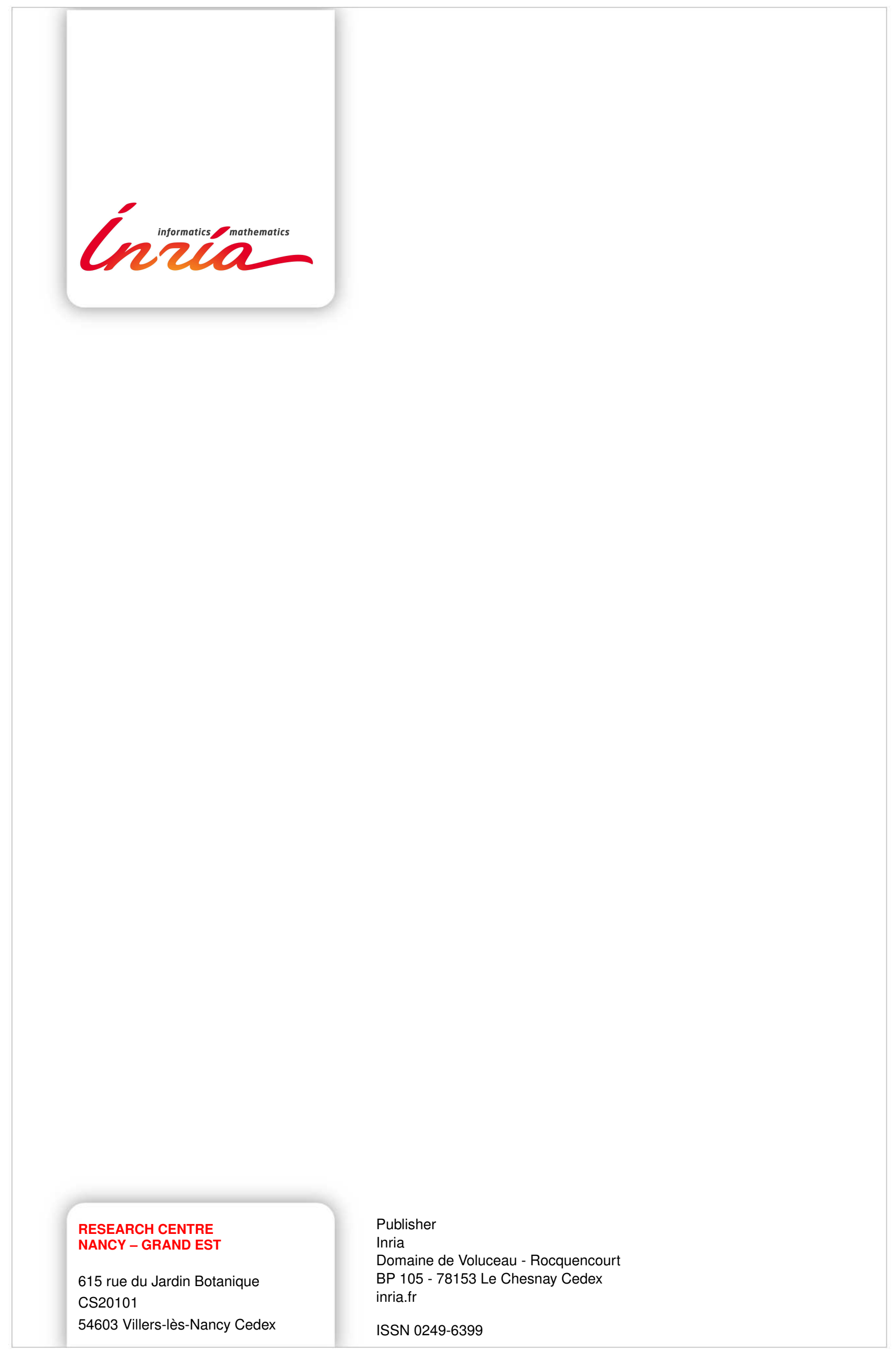

